\title{
NGC 4102: HIGH-RESOLUTION INFRARED OBSERVATIONS OF A NUCLEAR STARBURST RING
}

\author{
Sara C. BeCK ${ }^{1,4}$, John H. LaCY ${ }^{2,4}$, and Jean L. Turner ${ }^{3}$ \\ ${ }^{1}$ Department of Physics and Astronomy, Tel Aviv University, Ramat Aviv, Israel; sara@wise.tau.ac.il \\ ${ }^{2}$ Department of Astronomy, University of Texas at Austin, Austin, TX 78712, USA \\ 3 Department of Physics and Astronomy, UCLA, Los Angeles, CA 90095-1547, USA \\ Received 2010 June 10; accepted 2010 August 22; published 2010 September 27
}

\begin{abstract}
The composite galaxy NGC 4102 hosts a LINER nucleus and a starburst. We mapped NGC 4102 in the $12.8 \mu \mathrm{m}$ line of [Ne II], using the echelon spectrometer TEXES on the NASA IRTF, to obtain a data cube with 1".5 spatial, and $25 \mathrm{~km} \mathrm{~s}^{-1}$ spectral, resolution. Combining near-infrared, radio, and the [Ne II] data shows that the extinction to the starburst is substantial, more than $2 \mathrm{mag}$ at the $K$ band, and that the neon abundance is less than half solar. We find that the star formation in the nuclear region is confined to a rotating ring or disk of $4^{\prime \prime} .3(\sim 300$ pc) diameter, inside the inner Lindblad resonance. This region is an intense concentration of mass, with a dynamical mass $\sim 3 \times 10^{9} M_{\odot}$, and of star formation. The young stars in the ring produce the [Ne II] flux reported by Spitzer for the entire galaxy. The mysterious blue component of line emission detected in the near-infrared is also seen in [Ne II]; it is not a normal active galactic nucleus outflow.
\end{abstract}

Key words: galaxies: individual (NGC 4102) - galaxies: kinematics and dynamics - galaxies: starburst

\section{INTRODUCTION}

As many as half of the nearby galaxies may host LINERS, nuclei with distinctive low-ionization spectra that may be a distinct form of active galactic nucleus (AGN) or an extraordinary starburst (Ho et al. 1997). Many of these galaxies also contain intense starbursts in their cores. These "composite" galaxies have AGN+Starburst or LINER+Starburst nuclear spectra. How do the starburst and the active nucleus in these galaxies relate to each other? Does the AGN trigger the starburst, or does the starburst pour fuel into the AGN? These sources are hard to observe; high extinctions within galactic nuclei mean that optical and near-infrared data can be unreliable. Further, to distinguish between all the forms of activity that may occur in the small volume of a composite galaxy core requires high spatial and spectral resolution.

We report here on high spectral resolution mid-infrared [Ne II] observations of NGC 4102, a local composite galaxy that is close enough that we can potentially resolve structures within the nucleus. NGC 4102 is an Sb galaxy in the Ursa Major group. Its Galactic standard of rest redshift is $918 \mathrm{~km} \mathrm{~s}^{-1}$, for a Hubble distance of $14.3 \mathrm{Mpc}$; the best distance from the Tully-Fisher relation is $17 \mathrm{Mpc}$ (Tully et al. 2008), which we use. It has a $60 \mu \mathrm{m} / 100 \mu \mathrm{m}$ flux ratio of 0.71 , which is typical of spiral starbursts. CO observations of NGC 4102 (Jogee et al. 2005) show that its center is rich in molecular gas, with a derived $M_{\mathrm{H} 2}$ $(R<300 \mathrm{pc}) \sim 2.2 \times 10^{8} M_{\odot}$ (corrected to conversion factor $X_{\mathrm{CO}}=0.9 \times 10^{20} \mathrm{~cm}^{-2}\left(\mathrm{~K} \mathrm{~km} \mathrm{~s}^{-1}\right)^{-1}$ and $\left.17 \mathrm{Mpc}\right)$ and a central star formation rate (estimated from the non-thermal radio continuum) of $7 M_{\odot} \mathrm{yr}^{-1}$. The optical spectrum of NGC 4102 resembles that of a giant H II region (Ho et al. 1997), but it is not a simple pure starburst; its spectrum is transitional between LINERS and starbursts (Dudik et al. 2005) and it has a bright point-source nucleus. Near-infrared lines show a blue component at $\Delta v \sim-900 \mathrm{~km} \mathrm{~s}^{-1}$, relative to the galaxy (Roussel et al. 2003), which has been assumed to be a powerful outflow. It

\footnotetext{
4 Visiting Astronomer at the Infrared Telescope Facility, which is operated by the University of Hawaii under Cooperative Agreement no. NNX-08AE38A with the National Aeronautics and Space Administration, Science Mission Directorate, Planetary Astronomy Program.
}

is therefore classed as a composite galaxy that hosts both a starburst and a mildly active nucleus of LINER or Sy2 type.

NGC 4102 has often been observed in the near and middle infrared and radio, but mostly with relatively low spatial and spectral resolution. Because it is composite, at least two distinct forms of nuclear activity (starburst and whatever that point nucleus is) will be blended in observations with worse than $\sim 1^{\prime \prime}$ resolution. They have certainly been blended in the Spitzer Infrared Spectrograph (IRS) spectra, which have pixel sizes comparable to the entire nuclear region and slit sizes even larger. High spectral resolution can kinematically separate the different components of a galaxy, but the spectral resolution of the Spitzer IRS was not sufficient to distinguish the blue component or any smaller velocity offsets within the starburst region.

This complex nature of galactic centers demand observations with high spectral and spatial resolution. We report here on mid-infrared observations with arcsecond spatial and $25 \mathrm{~km} \mathrm{~s}^{-1}$ spectral resolution of the central region of NGC 4102.

\section{OBSERVATIONS}

\subsection{TEXES [Ne II] Data Cube}

The fine-structure line of $\mathrm{Ne}^{+}$at $12.8 \mu \mathrm{m}$ is usually one of the strongest mid-infrared emission lines in $\mathrm{H}$ II regions (Ho \& Keto 2007). [Ne II] is an excellent tracer of the ionized gas in $\mathrm{H}$ II regions because it is strong (stronger even than the infrared $\mathrm{H}$ I recombination lines), at a wavelength which is little affected by extinction for $A_{v} \precsim 40 \mathrm{mag}$, and has such a high critical density $\left(7.7 \times 10^{5} \mathrm{~cm}^{-3}\right)$ that collisional de-excitation is unlikely to be significant in $\mathrm{H}$ II regions. It is also an excellent kinematic probe; it is superior to the $\mathrm{H}$ I recombination lines for this purpose because the high atomic weight of neon makes the line less broadened by thermal motions. [Ne II] has been used to trace the kinematics and spatial distribution of ionized gas in Galactic $\mathrm{H}$ II regions and starburst galaxies (Zhu et al. 2008; Achtermann \& Lacy 1995; Elmegreen et al. 2009; AlonsoHerrero et al. 2009, and references therein).

We observed [Ne II] in NGC 4102 with TEXES (the Texas Echelon Cross Echelle Spectrograph; Lacy et al. 2002) on the NASA IRTF on Mauna Kea, on the night of 2009 June 1. TEXES 


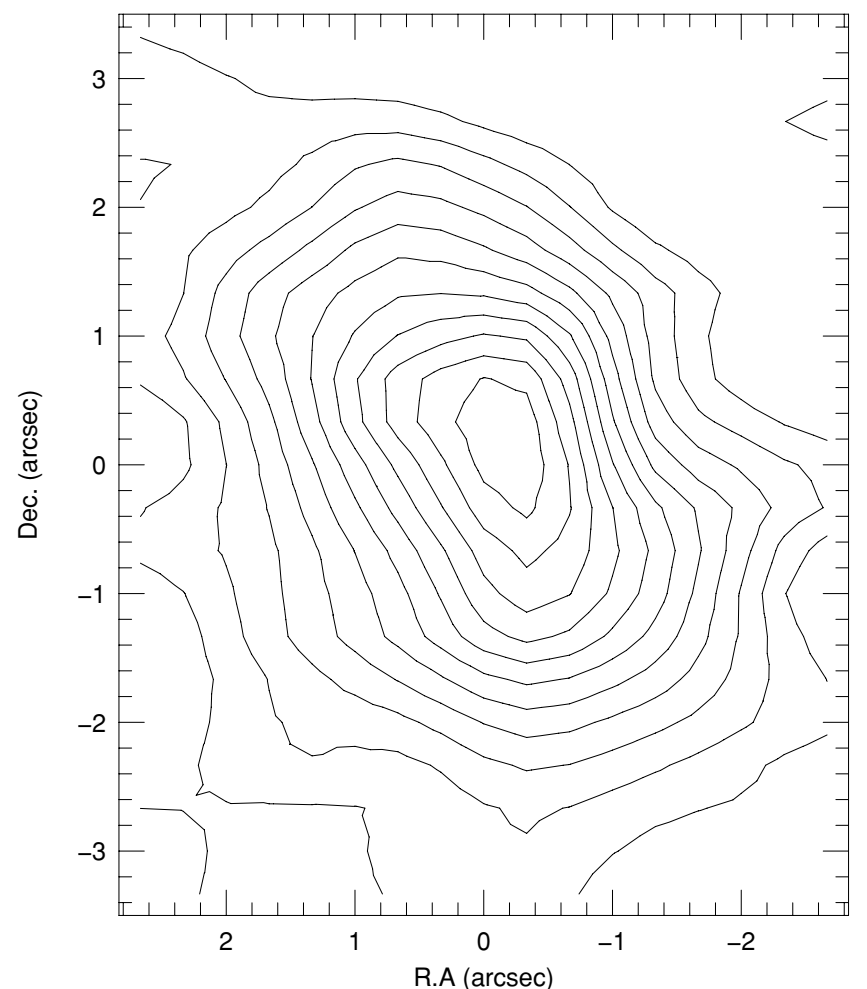

Figure 1. Integrated [Ne II] line flux. The data were interpolated between slit positions to give $\frac{1}{3}$ " sampling in both R.A. and decl. Resolution was seeing and diffraction limited to $\sim 1$ 1".4. Contours are linear and are integral multiples of $1.26 \times 10^{-3} \mathrm{erg} \mathrm{s}^{-1} \mathrm{~cm}^{-2} \mathrm{sr}^{-1}$.

is a sensitive spectrometer for the $5-25 \mu \mathrm{m}$ region with three resolution modes: these data were obtained in the mediumresolution long-slit mode, which gives spectral resolving power $R \sim 12,000$. The slit was $1^{\prime \prime} .5 \times 45^{\prime \prime}$ and the plate scale was $0.36 \times 8.9 \mathrm{~km} \mathrm{~s}^{-1}$ per pixel. The slit, which was oriented NS, was stepped across the galaxy in $1^{\prime \prime}$ increments, first west and then back east. There were no obvious inconsistencies between the two spectra at each position, giving us confidence in the telescope tracking. At each position, the galaxy was nodded $20^{\prime \prime}$ along the slit. The beams were subtracted and the spectra were added together to create a data cube. The velocities quoted are heliocentric.

The total emission of the [Ne II] line at each position is found by collapsing the data cube to produce the map in Figure 1. The spectrum at each point on the sky is shown in Figure 2, and the average spectrum over the entire emission region is in the upper right corner of Figure 2. The rest frequency of [Ne II] is $780.42 \mathrm{~cm}^{-1}(12.81 \mu \mathrm{m}$; Kelly \& Lacy 1995$)$ so the line frequency at the nominal velocity of NGC 4102 would be $778.23 \mathrm{~cm}^{-1}(12.85 \mu \mathrm{m})$. The [Ne II] emission extends over about $450 \mathrm{~km} \mathrm{~s}^{-1}$, Full Width Zero Intensity (FWZI), and is roughly centered on the nominal galactic velocity, although it is difficult to set the baseline because there are strong telluric features at 779.58 and $777.25 \mathrm{~cm}^{-1}$, the ends of the range, which cause very strong noise. The FWHM is $\sim 150 \mathrm{~km} \mathrm{~s}^{-1}$ in the strongest position.

\subsection{Archival Data}

We have obtained from the literature and data archive images of NGC 4102 at $\mathrm{Pa} \alpha$ and the $1.6 \mu \mathrm{m} \mathrm{J}$-band continuum from the Hubble Space Telescope (HST) and $3.6 \mathrm{~cm}$ radio continuum

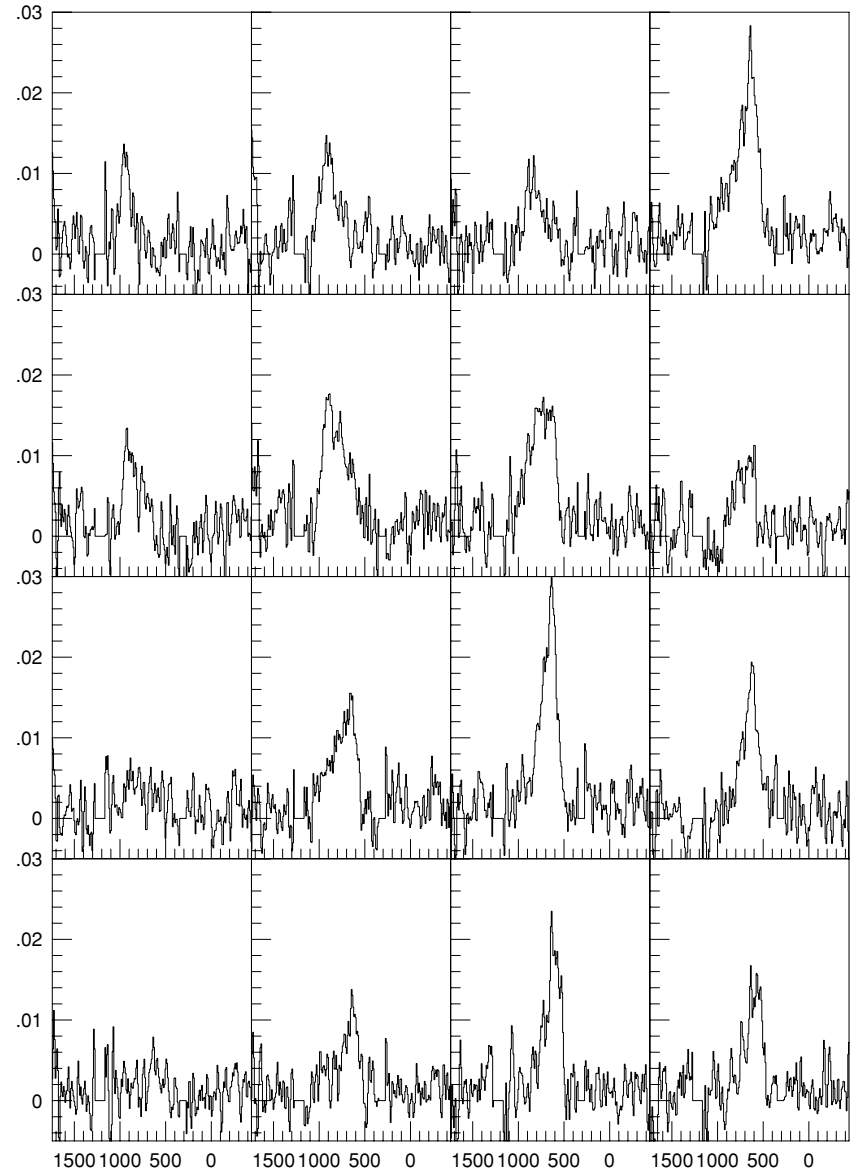

Figure 2. [Ne II] spectrum at each point on a $1^{\prime \prime}$ grid covering the central region of the previous figure. The center of the grid is at $(0,0)$ in the previous figure, north is up, and east is to the left. The $x$-axis is the heliocentric velocity and the $y$-axis units are $\mathrm{erg}\left(\mathrm{s} \mathrm{cm}^{2} \mathrm{~cm}^{-1} \mathrm{sr}\right)^{-1}$. The spectra are blanked to 0 across the strong atmospheric lines. The spectrum in the top right corner represents the integrated line shape of the galaxy; it was obtained by summing the individual spectra, weighted by the signal in the [Ne II] line, and is on an arbitrary flux scale.

from the VLA ${ }^{5}$ archival program AW416. The radio and $\mathrm{Pa} \alpha$ maps are shown in Figure 3 and are quite different from each other. The radio shows two strong peaks against a background of extended emission. The extended emission has no obvious ring structure but is a smooth plateau. The near-infrared maps have a weak point source at the nucleus and an inclined ring of emission, which is bright on the SW end and weak to the point of incompleteness on the east. The only alignment of the maps that gets the bright sources to agree is to identify the northern radio source with the galactic nucleus and the southern radio source with the bright SW peak in the near-infrared maps. This requires a shift of about $1^{\prime \prime}$ in each direction, marginally consistent with the quoted astrometrical accuracy of NICMOS.

We compare our [Ne II] results to data taken with the Short High (SH) module of the IRS on the Spitzer spacecraft, in PID 30745. The positions used for the IRS slits agree with the radio maps and the entire radio emission region falls into the slit of the SH module in which the [Ne II] line is observed. If the nominal, unshifted coordinates are used for $\mathrm{Pa} \alpha$ the galactic nuclear region is only half in the SH module slit. This further justifies the sizable shift we apply to the $\mathrm{Pa} \alpha$ image.

\footnotetext{
5 The National Radio Astronomy Observatory is a facility of the National Science Foundation operated under cooperative agreement by Associated Universities, Inc.
} 

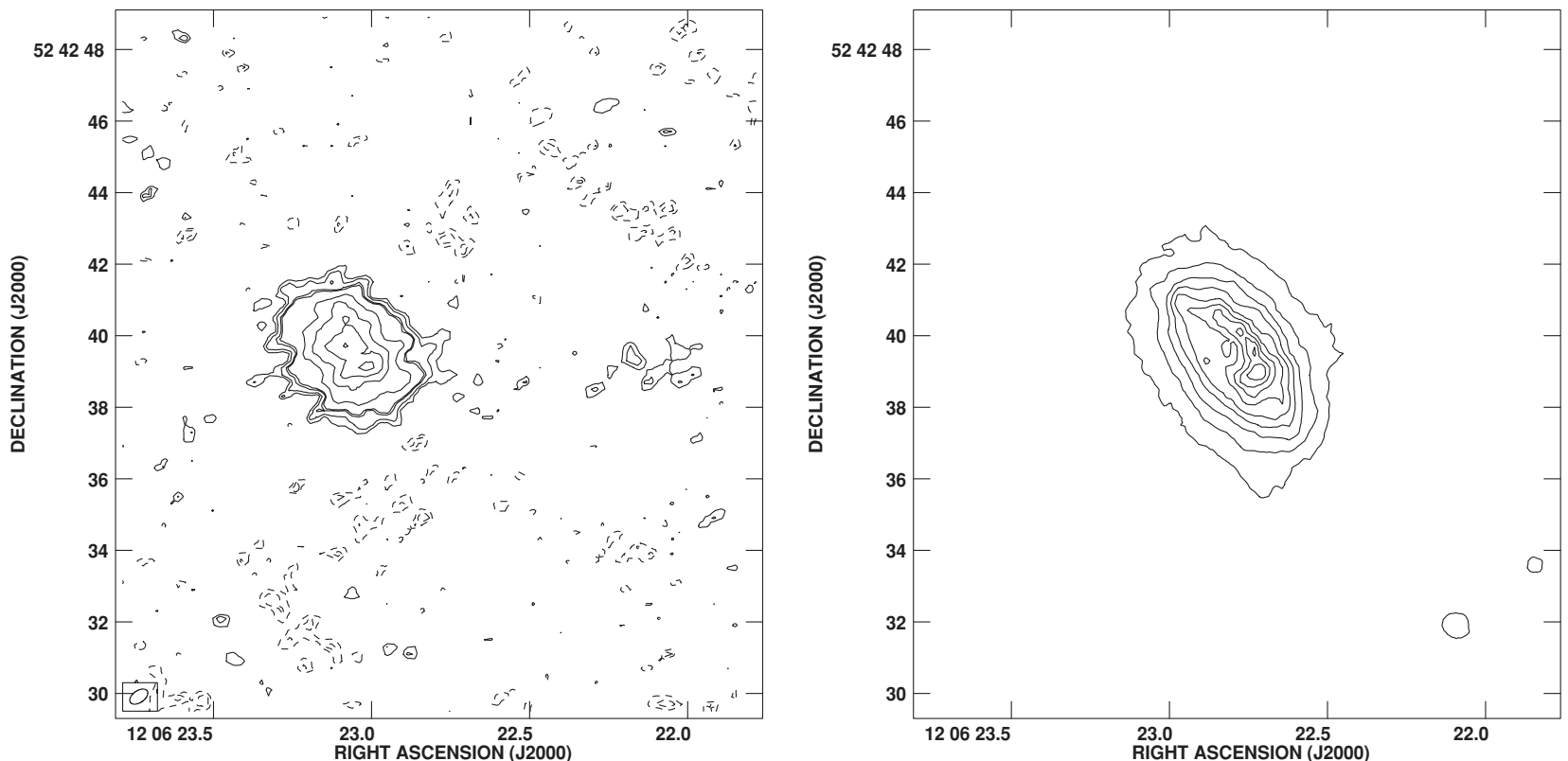

Figure 3. Left: NGC 4102 at $3.6 \mathrm{~cm}$ from the archival VLA program AW416. The $3.6 \mathrm{~cm}$ beam is shown in the bottom left corner. The radio map contour intervals are $\pm 2^{N / 2}$ and the contour level is $0.18 \mathrm{mJy} \mathrm{bm}^{-1}$. Right: NGC 4102 in the Pa $\alpha$ line from the $H S T$ archive. The contour level is $1 \%$ of the peak and contour intervals are $1,4,9, \ldots, 100$. Note the well-defined asymmetric ring of the near-infrared. The apparent offset of the two images is discussed in the text.

\section{TOTAL FLUX, ABUNDANCE, AND EXTINCTION}

We recover a total flux of [Ne II] by summing the emission over the $1 \mathrm{~cm}^{-1}$ wavelength and $4^{\prime \prime} \times 6^{\prime \prime}$ spatial range in which the line was seen. The wavelength window included in this calculation corresponds to a full velocity extent of only $385 \mathrm{~km} \mathrm{~s}^{-1}$, typical of the velocity ranges in star-forming galaxies and excluding the very high velocities associated with AGNs. The total emission is $2.2 \times 10^{-15} \mathrm{~W} \mathrm{~m}^{-2}$, with an estimated error, largely due to the weak and noisy continuum, of $\pm 20 \%$. This corresponds to $7.3 \mathrm{Jy}$ at the $R=600$ resolution of the IRS, in excellent agreement with the total $7.5 \mathrm{Jy}$ found for this line by Spitzer. This shows that (1) the entire Spitzer-observed emission is confined to the inner 5" (about 2 pixels of the Spitzer detector array) and (2) the entire Spitzer-observed emission is at low, starburst-like, velocity; there is no AGN contribution to the [Ne II] line.

Total [Ne II] flux depends on the abundance of neon and the fractional abundance of $\mathrm{Ne}^{+}$. $\mathrm{Ne}^{+}$is created at the relatively low energy of $21.5 \mathrm{eV}$, and most of the neon in starbursts is in this ionization state. AGNs usually create more $\mathrm{Ne}^{+2}$; the ratio of [Ne II] to the higher-excitation lines can be used to separate starburst from non-stellar galactic nuclei (Sturm et al. 2006). Spitzer IRS spectra of NGC 4102 show a relatively weak [Ne III] line of about $0.7 \mathrm{Jy}$, which includes any contribution from the AGN. We conclude that almost all the neon in this region is in the $\mathrm{Ne}^{+}$state. So the observed [Ne II] flux, the observed $\mathrm{Br} \gamma$ flux of $6.0 \times 10^{-17} \mathrm{~W} \mathrm{~m}^{-2}$ (Roussel et al. 2003), and the total $3.6 \mathrm{~cm}$ flux of $34 \mathrm{mJy}$ in a $3^{\prime \prime} .75$ box in the center of the galaxy are consistent with neon abundance of 0.4 solar and $A_{K}=$ 2.2 mag. (Neon parameters are discussed in Dinerstein et al. 2002.) This would imply $A_{12.8 \mu \mathrm{m}}$ of $0.6 \mathrm{mag}$ and $A_{\mathrm{Pa} \alpha}$ higher by about $0.5 \mathrm{mag}$ than the $2.4 \mathrm{mag}$ found by Roussel et al. (2003). The discrepancy is probably because the extinction is so high that the near-infrared wavelengths do not see though the nucleus. The line ratios would agree with a lower extinction and higher neon abundance if the radio flux contains a significant non-thermal component. The galactic nucleus, the nature of whose radio emission is unknown, does contribute about $10 \%$ of the total $3.6 \mathrm{~cm}$ emission, and there may be other non-thermal sources in the region.

\section{SPATIAL STRUCTURE OF THE EMISSION FROM NEAR-INFRARED TO RADIO}

The near-infrared and radio maps each show a peak at the nucleus and one in the SW. The SW is stronger than the nucleus in each case, although the contrast is less in the radio than in Pa $\alpha$. In the radio map the SW source looks like an independent feature, perhaps a very bright star cluster, rather than the limb-brightened region of the annular emission its infrared appearance suggests. The total [Ne II] emission, shown in Figure 1, appears to be in a disk or ring with outer diameter about 4.3 , about 300 pc. Unlike both the near-infrared and radio, the [Ne II] does not show the two distinct sources. The line emission is stronger in the southern part of the galaxy, but unlike the near-infrared the intensity varies smoothly; this may be the effect of the lower spatial resolution in the mid-infrared. We believe that the ring seen in the near-infrared map does not appear in the [Ne II] map because the structure is on too small a scale; the [Ne II] spatial resolution was seeing and diffraction limited to about $1^{\prime \prime}-1$."4.

The $\mathrm{Pa} \alpha$ line, [Ne II] line, and $3.6 \mathrm{~cm}$ radio continuum all map ionized gas; why are their distributions so different? The disagreement between the [Ne II] and $\mathrm{Pa} \alpha$ distribution probably shows the influence of extinction on the near-infrared image; extinction at $\mathrm{Pa} \alpha$ is about 10 times higher than that at [Ne II]. But the extinction at [Ne II] is small, as shown above, so the difference between the mid-infrared and radio images is not likely to be due to obscuration. While the central AGN source is presumably dominated by non-thermal emission, the extended $3.6 \mathrm{~cm}$ radio emission in the starburst should be mostly thermal and indeed it is extended and resembles the [Ne II].

Our high-resolution spectra are not well suited for continuum measurements and we detect the $12.8 \mu \mathrm{m}$ continuum only weakly. We can say, however, that the $12.8 \mu \mathrm{m}$ continuum does not resemble the near-infrared continuum; it is not noticeably stronger in the south, nor is there a strong mid-infrared contin- 


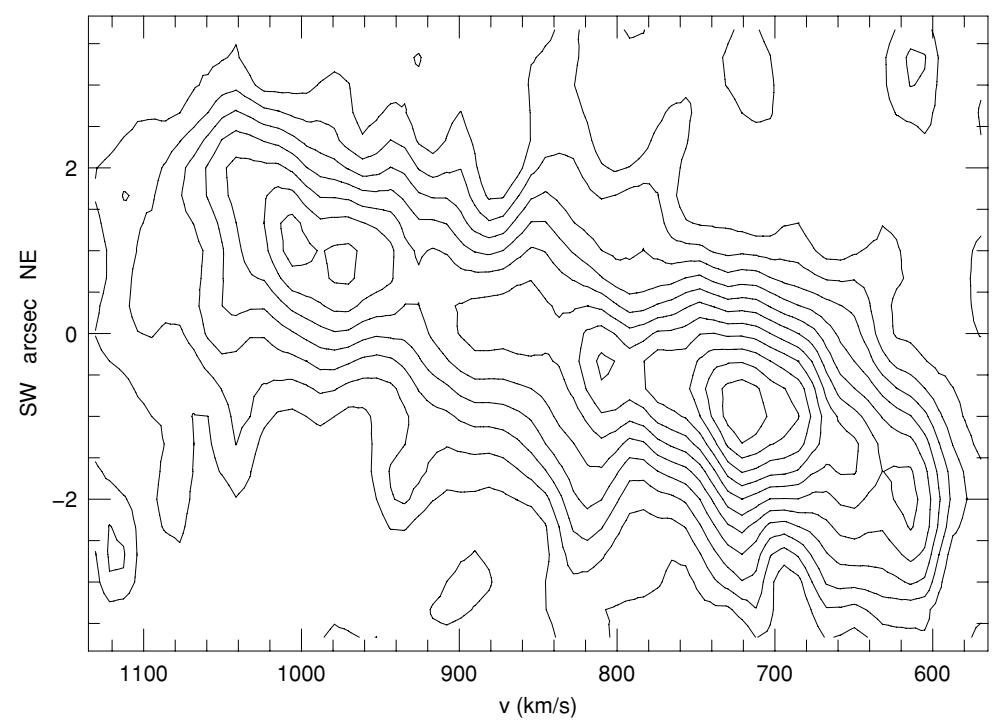

Figure 4. Position-velocity diagram taken down the major axis of the galaxy $\left(45^{\circ}\right)$. Contour levels are $2,4,6, \ldots \times 10^{-3} \mathrm{erg} \mathrm{s}^{-1} \mathrm{~cm}^{-2} \mathrm{sr}^{-1}$. The data have been interpolated spatially onto a 0!'333 grid oriented along the galactic major and minor axes, summed along the minor axis, and then smoothed spectrally and spatially by 2 pixels.

uum source associated with the SW radio source. This probably reflects the range of extinctions, dust abundances, and temperatures in the $\mathrm{H}$ II regions around the starburst.

\subsection{The SW Radio Source: A Luminous Super Star Cluster?}

The nature of the radio source at R.A. $12^{\mathrm{h}} 06^{\mathrm{m}} 23.0$, decl. $52^{\circ} 42^{\prime} 39^{\prime \prime} .1$ is not clear. It is strong at $3.6 \mathrm{~cm}$ and does not appear in $6 \mathrm{~cm}$ and $20 \mathrm{~cm}$ archival maps, which argues that it is mostly thermal. There is the possibility that the 6 and $20 \mathrm{~cm}$ maps do not show the SW source because it is too small and their beams were larger than 1..7; this could be confirmed or disproved with high-resolution observations at longer wavelengths (as in, e.g., Beck et al. 2000). In the meantime, we will adopt the most straightforward conclusion that the SW source is mostly thermal: a bright giant $\mathrm{H}$ II region in the starburst ring. Then its radio flux at $3.6 \mathrm{~cm}$ of $3 \mathrm{mJy}$ requires, for standard assumptions of density and $T_{e}$, an ionization of about $8.9 \times 10^{52} \gamma \mathrm{s}^{-1}$. This is equivalent to $8.9 \times 10^{3}$ "equivalent O7 stars" (Vacca 1994) in a region no larger than $0.41 \times 0$.'17, or around $34 \times 14 \mathrm{pc}$. We used STARBURST99 with continuous star formation and ages 3-5 Myr to calculate the mass of stars associated with such ionization, and obtained masses of 2-3 $\times 10^{6} M_{\odot}$ for a Kroupa initial mass function (IMF) to $4.5 \times 10^{6} M_{\odot}$ for a Salpeter IMF. If this is a single Super Star Cluster or young Globular Cluster recently formed in the starburst ring, it is the largest to be observed in a starburst ring (Kormendy \& Kennicutt 2004) and among the most massive and luminous anywhere in the local universe: it is very similar to the central source of NGC 5253 (Turner \& Beck 2004), the largest well-studied single embedded star cluster, and within factors of a few of the immense double cluster in the nearby LIRG IRAS 04296+2923 (Meier et al. 2010). This source might, in another setting, be counted as a dwarf galaxy in its own right; it is as large as many of the tidal dwarfs that form in the debris of strong interactions (Sheen et al. 2009, and references therein).

\section{KINEMATICS AND MASS}

\subsection{A Rotating Ring}

That the ionized gas in NGC 4102 is rotating may be seen clearly in the spectra of Figure 2 as the line peak shifts from position to position. A position-velocity diagram of the [Ne II] emission along the major axis of the galaxy is shown in Figure 4. It is consistent with rotation, with some evidence for non-circular motion that will be discussed in the following section. In spatial appearance the [Ne II] could be a disk or a ring. There are two reasons we think it is more likely to be a ring: (1) the near-infrared maps show clear gas depletion in the center and (2) a rotating disk of gas would reach much higher velocities at small radii (although possibly over an undetectably small area). So the [Ne II] observations indicate that NGC 4102 hosts a rotating nuclear starburst ring. The velocity gradient is $135 \mathrm{~km} \mathrm{~s}^{-1} \operatorname{arcsec}^{-1}$, or $2.0 \mathrm{~km} \mathrm{~s}^{-1} \mathrm{pc}^{-1}$, over the inner $3^{\prime \prime}$ or 200 pc diameter, measured from Figure 4.

Jogee \& Kenney (1996) report CO measurements with a $2^{\prime \prime}$ beam. NGC 4102 has a bar and lens, and gas motions in the nuclei of barred galaxies are often non-circular; the $\mathrm{CO}$ measurements clearly show a turnover in the rotation curve at radii greater than $3^{\prime \prime}$, which Jogee \& Kenney (1996) attribute to gas streaming along a bar. The [Ne II] emission is more limited than that of the $\mathrm{CO}$, covering only $\sim 2$ of the $\mathrm{CO}$ beams and not extending to the region where the $\mathrm{CO}$ rotation curve turns over. The velocity gradient of the $\mathrm{CO}$ is $\sim 83 \mathrm{~km} \mathrm{~s}^{-1} \operatorname{arcsec}^{-1}$, or $1.2 \mathrm{~km} \mathrm{~s}^{-1} \mathrm{pc}^{-1}$, over the inner 4".5 (300 pc diameter) measured from Figure 1 of Jogee \& Kenney (1996). That the [Ne II] emission has a greater velocity gradient than the $\mathrm{CO}$, but a very similar velocity range and even a similar distribution of brightness with velocity, indicates that the $\mathrm{CO}$ and [Ne II] emitting regions are not spatially identical and that the ionized gas, and thus the young stars, lie along the inner edge of the molecular gas ring. This may be consistent with models for star formation in the presence of shear (Maciejewski 2008); observations of the molecular gas with higher spatial resolution are needed to clarify the situation.

Assuming circular orbits and an inclination angle of $58^{\circ}$ (Verheijen \& Sancisi 2001), we calculate the total mass inside the full $135 \mathrm{pc}$ of the $\left[\mathrm{Ne}\right.$ II] ring to be $3 \times 10^{9} M_{\odot}$. The $\mathrm{CO}$, which had a less steep slope over a larger distance, gives a dynamical mass of $2.8 \times 10^{9} M_{\odot}$ for a radius of $\sim 187 \mathrm{pc}$.

Our central mass for NGC 4102 is very similar to the masses inferred for the central $\sim 100$ pc regions of nearby galaxies as 
diverse as M31, NGC 4258, and our own Galactic center (Burton \& Gordon 1978; Sofue \& Rubin 2001).

\subsection{A High-velocity Outflow?}

The one velocity feature that does not fit the simple picture of a rotating ring is the weak feature at $781.0 \mathrm{~cm}^{-1}(12.80 \mu \mathrm{m})$. This is blueshifted $200 \mathrm{~km} \mathrm{~s}^{-1}$ from the rest velocity of [Ne II] and $\sim 960 \mathrm{~km} \mathrm{~s}^{-1}$ from the velocity of the galaxy. We believe it to be real because it appears in several positions and agrees in velocity with a blueshifted feature in the near-infrared $\mathrm{H}$ I lines of $\mathrm{Pa} \alpha$ and $\mathrm{Br} \gamma$ (Roussel et al. 2003). The blue component is an estimated one-third of the near-infrared $\mathrm{Pa} \beta$ and $\mathrm{Br} \gamma$ fluxes, but this does not scale to the mid-infrared, where we estimate that the blue component is less than $10 \%$ of the peak of the [Ne II] line (integrated over the same slit). When this feature was detected in the near-infrared, Roussel et al. (2003) called it an outflow and assumed that the red side could not be seen because the extinction is higher to the red or far side of the system. That explanation is less satisfactory in light of the [Ne II] data. First, we see in the $[\mathrm{Ne} \mathrm{II}]$ spectra that the blue feature is very narrow. Its FWHM is hard to determine because of the low signal-tonoise ratio but is clearly less than $150 \mathrm{~km} \mathrm{~s}^{-1}$. AGN outflows are usually broader by more than a factor of 10 . For an outflow to have such a narrow velocity spread, it must have a very narrow opening angle and be more like a jet than a cone. But there is no other evidence for a jet in NGC 4102. Second, if the absence of the redshifted feature in the near-infrared feature is in fact due to the higher extinction to the red or far side of the outflow, it may be expected to be detectable at $12.8 \mu \mathrm{m}$ where the extinction is so much lower than at the near-infrared. There are $2 \sigma$ wiggles around $776.2 \mathrm{~cm}^{-1}(12.88 \mu \mathrm{m})$, the wavelength equidistant to the red, but they are not convincing.

We have at present no really satisfactory explanation of the blueshifted feature. If it is an outflow, and the red side is not detected for extinction or another reason, it is a very unusual one. If it is due to the unlikely coincidence of a foreground object, no such object appears at any wavelength yet observed and NGC 4102 is far out of the galactic plane. The blue feature is spatially extended and is detected at several positions north of the nucleus, so it cannot be associated with the SW radio source. There are no features at that velocity (or the red equivalent) in the $\mathrm{CO}$ position-velocity diagrams, and we know of no emission line near that wavelength.

\section{CONCLUSIONS}

The high-resolution [Ne II] observations of NGC 4102 have significantly clarified the starburst underway in that galaxy: it is located in a rotating ring, about $400 \mathrm{pc}$ in diameter, consistent with the inner Lindblad resonance. The starburst covers roughly the inner third of the rotating molecular disk observed in CO. The entire [Ne II] flux observed by Spitzer can be attributed to this starburst ring. The starburst produces a total ionization of about $9.9 \times 10^{53} \gamma^{-1}$, equal to $9.9 \times 10^{4}$ standard O7 stars, and a total mass in young stars of $3-5 \times 10^{7} M_{\odot}$ depending on IMF and starburst age. The relative strengths of the near-infrared continuum, radio continuum, and [Ne II] line vary around the ring, mostly due, we believe, to extinction effects. The most notable structure is a peak in the radio emission SW of the nucleus which, if it is a single embedded star cluster, is as bright as the largest and brightest known. Observations with even higher resolution could perhaps resolve this and other structures in the ring.
We obtain a dynamical mass of $M(R<164 \mathrm{pc})=3 \times 10^{9} M_{\odot}$ for the inner $164 \mathrm{pc}$ radius, compared to $M(R<250 \mathrm{pc})=$ 5-6 $\times 10^{9} M_{\odot}$ found from the CO results. Thus, about $1 \%$ of the dynamical mass of this region is in young stars. The mass appears to grow with radius as $M \propto r^{1.8}$.

NGC 4102 contains a mild active nucleus as well as a starburst. When a nuclear starburst is found in the same galaxy with an AGN, it raises the possibility that the AGN has triggered the starburst somehow. But there is no evidence for AGN influence on the NGC 4102 starburst. This star formation episode most likely reflects the secular evolution of the galaxy. The center of NGC 4102 is full of molecular gas streaming in; enhanced star formation in such a region is "almost inevitable" (Kormendy \& Kennicutt 2004).

The only possible sign of nuclear activity in the infrared is the high-velocity emission seen in the hydrogen lines and in [Ne II]. The cause of this velocity feature is still not known: if it is an AGN outflow it is a very usual one, but if it is a foreground object it is not easy to explain.

We thank Matt Richter and the TEXES team for obtaining the data. TEXES observations at the IRTF are supported by NSF AST-0607312 and AST-0708074 (to Matt Richter). Part of this paper was based on archival data from the VLA of The National Radio Astronomy Observatory, which is operated by Associated Universities, Inc., under cooperative agreement with the National Science Foundation. This research has made use of the NASA \& IPAC Extragalactic Database (NED) which is operated by the Jet Propulsion Laboratory, Caltech, under contract with NASA. We thank the referee for helpful comments, and S.C.B. thanks M. Shull and the University of Colorado for hospitality while this was written.

\section{REFERENCES}

Achtermann, J. M., \& Lacy, J. H. 1995, ApJ, 439, 163

Alonso-Herrero, A., et al. 2009, ApJ, 697, 660

Beck, S. C., Turner, J., \& Kovo, O. 2000, AJ, 120, 244

Burton, W. B., \& Gordon, M. A. 1978, A\&A, 63, 7

Dinerstein, H., Richter, M., Lacy, J. H., \& Sellgren, K. 2002, AJ, 125, 265

Dudik, R. P., Satyapal, S., Gliozzi, M., \& Sambruna, R. M. 2005, ApJ, 620, 113

Elmegreen, B., Galliano, E., \& Alloin, D. 2009, ApJ, 703, 1297

Ho, L., Fillipenko, A., \& Sargent, W. 1997, ApJS, 112, 315

Ho, L. C., \& Keto, E. 2007, ApJ, 658, 314

Jogee, S., \& Kenney, J. D. P. 1996, in ASP Conf. Ser. 91, Barred Galaxies, ed.

R. Buta, D. A. Crocker, \& B. G. Elmegreen (San Francisco, CA: ASP), 230 Jogee, S., Scoville, N., \& Kenney, J. D. P. 2005, ApJ, 630, 837

Kelly, D. M., \& Lacy, J. H. 1995, ApJ, 464, L161

Kormendy, J., \& Kennicutt, R. C. 2004, ARA\&A, 42, 603

Lacy, J., Richter, M., Greathouse, T., \& Zhu, Q.-F. 2002, PASP, 114, 153

Maciejewski, W. 2008, in IAU Symp. 245, Formation and Evolution of Galaxy Bulges, ed. M. Bureau, E. Athanassoula, \& B. Barbuy (Cambridge: Cambridge Univ. Press), 161

Meier, D. S., Turner, J. L., Beck, S. C., Gorjian, V., Tsai, C. W., \& Van Dyck, S. 2010, AJ, in press

Roussel, H., Helou, G., Beck, R., Condon, J. J., Bosman, A., Matthews, K., \& Jarrett, T. 2003, ApJ, 593, 733

Sheen, Y.-K., et al. 2009, AJ, 138, 1911

Sofue, Y., \& Rubin, V. 2001, ARA\&A, 39, 137

Sturm, E., et al. 2006, ApJ, 653, L13

Tully, R., Shaya, E., Karachentsev, I. D., Courtois, E., Kocevski, D., Rizzi, L., \& Peel., A. 2008, ApJ, 676, 184

Turner, J. L., \& Beck, S. C. 2004, ApJ, 602, L85

Vacca, W. D. 1994, ApJ, 421, 140

Verheijen, M. A. W., \& Sancisi, R. 2001, A\&A, 370, 765

Zhu, Q.-F., Lacy, J. H., Jaffe, D., Richter, M., \& Greathouse, T. 2008, ApJS, 177,584 